\title{
Initial Clinical Results of a Novel Immuno-PET Theranostic Probe in Human Epidermal Growth Factor Receptor 2-Negative Breast Cancer
}

\author{
Caroline Rousseau ${ }^{1,2}$, David M. Goldenberg ${ }^{3,4}$, Mathilde Colombié ${ }^{1}$, Jean-Charles Sébille ${ }^{1}$, Philippe Meingan ${ }^{5}$, \\ Ludovic Ferrer $^{2,6}$, Pierre Baumgartner ${ }^{7}$, Evelyne Cerato ${ }^{8}$, Damien Masson ${ }^{9}$, Mario Campone ${ }^{10}$, Aurore Rauscher ${ }^{7}$, \\ Vincent Fleury ${ }^{1}$, Catherine Labbe ${ }^{5}$, Alain Faivre Chauvet ${ }^{11}$, Jean-Sebastien Fresnel ${ }^{10}$, Claire Toquet $^{12}$, Jacques Barbet ${ }^{13}$, \\ Robert M. Sharkey ${ }^{3}$, Loic Campion ${ }^{2,14}$, and Françoise Kraeber-Bodéré1,2,11 \\ ${ }^{1}$ Nuclear Medicine, ICO Cancer Center, Nantes, France; ${ }^{2}$ CRCINA, University of Nantes, INSERM UMR1232, CNRS-ERL6001, \\ Nantes, France; ${ }^{3}$ Immunomedics, Inc., Morris Plains, New Jersey; ${ }^{4}$ IBC Pharmaceuticals, Inc., Morris Plains, New Jersey; \\ ${ }^{5}$ Radiology, ICO Cancer Center, Nantes, France; ${ }^{6}$ Physics, ICO Cancer Center, Nantes, France; ${ }^{7}$ Pharmacy, ICO Cancer Center, \\ Nantes, France; ${ }^{8}$ DRCI, University Hospital, Nantes, France; ${ }^{9}$ Biology Department, University Hospital, Nantes, France; ${ }^{10}$ Oncology, \\ ICO Cancer Center, Nantes, France; ${ }^{11}$ Nuclear Medicine, University Hospital, Nantes, France; ${ }^{12}$ Pathology Department, University \\ Hospital, Nantes, France; ${ }^{13}$ GIP Arronax, Saint-Herblain, France; and ${ }^{14}$ Biometrics, ICO Cancer Center, Nantes, France
}

This prospective study evaluated the imaging performance of a novel pretargeting immunologic PET (immuno-PET) method in patients with human epidermal growth factor receptor 2 (HER2)negative, carcinoembryonic antigen (CEA)-positive metastatic breast cancer, compared with CT, bone MRI, and ${ }^{18} \mathrm{~F}-\mathrm{FDG}$ PET. Methods: Twenty-three patients underwent whole-body immunoPET after injection of $150 \mathrm{MBq}$ of ${ }^{68} \mathrm{Ga}-I \mathrm{MP} 288$, a histamine-succinyl-glycine peptide given after initial targeting of a trivalent anti-CEA, bispecific, antipeptide antibody. The gold standards were histology and imaging follow-up. Tumor SUVs (SUV max $_{\text {ax }}$ and SUV $\left.V_{\text {mean }}\right)$ were measured, and tumor burden was analyzed using total tumor volume and total lesion activity. Results: The total lesion sensitivity of immuno-PET and ${ }^{18}$ F-FDG PET were $94.7 \%(1,116 / 1,178)$ and $89.6 \%(1,056 / 1,178)$, respectively. Immuno-PET had a somewhat higher sensitivity than CT or ${ }^{18} \mathrm{~F}-\mathrm{FDG}$ PET in lymph nodes (92.4\% vs. $69.7 \%$ and $89.4 \%$, respectively) and liver metastases ( $97.3 \%$ vs. $92.1 \%$ and $94.8 \%$, respectively), whereas sensitivity was lower for lung metastases (48.3\% vs. $100 \%$ and $75.9 \%$, respectively). Immuno-PET showed higher sensitivity than $\mathrm{MRI}$ or ${ }^{18} \mathrm{~F}-$ FDG PET for bone lesions (95.8\% vs. $90.7 \%$ and $89.3 \%$, respectively). In contrast to ${ }^{18} \mathrm{~F}-\mathrm{FDG}$ PET, immuno-PET disclosed brain metastases. Despite equivalent tumor $S U V_{\text {max }}, S_{U} V_{\text {mean, }}$ and total tumor volume, total lesion activity was significantly higher with immuno-PET than with ${ }^{18} \mathrm{~F}$ FDG PET $(P=0.009$ ). Conclusion: Immuno-PET using anti-CEAvantiIMP288 bispecific antibody, followed by ${ }^{68} \mathrm{Ga}-\mathrm{IMP} 288$, is a potentially sensitive theranostic imaging method for HER2-negative, CEA-positive metastatic breast cancer patients and warrants further research.

Key Words: breast cancer; immuno-PET; pretargeting; bispecific antibody; ${ }^{68} \mathrm{Ga}$

J Nucl Med 2020; 61:1205-1211

DOI: 10.2967/jnumed.119.236000

Received Sep. 6, 2019; revision accepted Dec. 4, 2019.

For correspondence or reprints contact: Caroline Rousseau, ICO Gauducheau Cancer Center, Boulevard Monod, 44805 Saint Herblain Cedex, France.

E-mail: c-rousseau@ico.unicancer.fr

Published online Mar. 13, 2020.

COPYRIGHT (C 2020 by the Society of Nuclear Medicine and Molecular Imaging.
B reast cancer $(\mathrm{BC})$ is the most common cancer among women worldwide, and about $40 \%$ of all $\mathrm{BC}$ patients have a recurrence (1). Imaging plays an important role in BC management, and PET using ${ }^{18} \mathrm{~F}$-FDG is recommended for relapse detection and therapy assessment (2). However, ${ }^{18} \mathrm{~F}$-FDG is not an oncology-specific tracer and does not allow selection of patients for hormonal or targeted therapies. Recently, ${ }^{18} \mathrm{~F}$-fluoroestradiol was approved for the characterization of estrogen receptor expression in known or suspected metastatic lesions of estrogen receptorpositive BC (3).

In the last decade, a promising option to improve diagnostic and theranostic imaging has been immunologic PET (immuno-PET), which combines the high sensitivity and quantitative capabilities of PET with the specificity and selectivity of a monoclonal antibody $(\mathrm{mAb})$ against a given tumor cell-surface marker. This combination potentially provides a noninvasive, in vivo-quantifiable, 3-dimensional whole-body tumor biomarker expression cartography, permitting tumor detection and patient monitoring prospectively (4). In BC, clinical studies have reported a good performance for immunoconjugates targeting human epidermal growth factor receptor 2 (HER2) radiolabeled with PET emitters having different half-lives $\left({ }^{68} \mathrm{Ga},{ }^{64} \mathrm{Cu}\right.$, and $\left.{ }^{89} \mathrm{Zr}\right)(5,6)$, and in preclinical studies, several biomarkers, such as syndecan-1, have been considered for triple-negative $\mathrm{BC}(7,8)$.

Carcinoembryonic antigen (CEA), specifically CEA cell-adhesion molecule-5, represents an attractive target in $\mathrm{BC}$, with serum CEA positivity (i.e., $\geq 5 \mathrm{ug} / \mathrm{L}$ ) being observed in $50 \%-60 \%$ of patients with metastatic disease (9), with a false-positive rate of $5 \%$ (10). A recent metaanalysis showed that elevated CEA was significantly associated with poorer disease-free survival and overall survival in BC, independent of tumor size, lymph node metastasis, or tumor grade and irrespective of estrogen receptor or HER2 status $(11,12)$. Elevated CEA was more related to the non-triple-negative and nonluminal tumor type and older age (12). Moreover, most invasive lobular, tubular, and cribriform carcinomas were CEA-negative (72\%). Conversely, $70 \%$ of invasive ductal carcinomas were CEA-positive (13). 
In the 1970s and later, the potential of cancer imaging using ${ }^{131} \mathrm{I}$ - and ${ }^{99 \mathrm{~m}} \mathrm{Tc}-$ labeled anti-CEA antibodies was reported for CEA-positive tumors, including BC, but the method was limited by the poor resolution of SPECT and the high blood activity observed with directly radiolabeled mAbs and fragments (14-17). Anti-CEA targeted therapies are still under development, and labetuzumab govitecan (anti-CEA cell-adhesion molecule-5/SN-38 antibody-drug conjugate) recently has shown promising efficacy with manageable toxicity in metastatic colorectal cancer patients (18). Durable objective responses in highly pretreated metastatic triple-negative $\mathrm{BC}$ patients, as well as in estrogen receptor-positive, HER2-negative $\mathrm{BC}$, were reported with an antibody-drug conjugate that targets the human trophoblast cell-surface antigen-2, suggesting the advance of a targeted drug conjugate platform for solid tumors $(19,20)$.

Pretargeting refers to a system of improved image contrast and high sensitivity obtained by first administering an antiCEA bispecific antibody (BsmAb), followed by haptens labeled with ${ }^{111} \mathrm{In}$ or ${ }^{131} \mathrm{I}(21-23)$. TF2 is an engineered trivalent BsmAb composed of a humanized anti-histamine-succinylglycine antigen-binding fragment derived from the murine 679 antibody and 2 humanized anti-CEA antigen-binding fragments (trivalency) of the humanized hMN-14 antibody, formed into a $157-\mathrm{kD}$ protein by the dock-and-lock procedure (24). The ${ }^{68} \mathrm{Ga}$-IMP288 bivalent peptide pretargeted with TF2 has demonstrated promising immuno-PET performance in a pilot optimization clinical trial (25). The current prospective study was designed to assess the initial sensitivity, safety, and preferred conditions of pretargeting immuno-PET using the BsmAb TF2 and the ${ }^{68} \mathrm{Ga}-\mathrm{IMP} 288$ peptide, in comparison to ${ }^{18} \mathrm{~F}$-FDG PET and conventional imaging in relapsed HER2-negative BC patients.

\section{MATERIALS AND METHODS}

\section{Population}

Patients at least $18 \mathrm{y}$ old, with progressive HER2-negative metastatic BC after standard treatments and presenting with a CEA serum level of at least $10 \mathrm{ng} / \mathrm{mL}$ and at least 1 lesion $10 \mathrm{~mm}$ or larger on conventional imaging (CT, MRI, and bone scans), were eligible. The other inclusion criteria were a Karnofsky performance status of at least 70, a minimum life expectancy of 6 mo, a creatinine level of no more than 2.5 times the normal level, and normal serum human antimouse antibody and human antihuman antibody (HAHA) titers. Women of child-bearing potential had to have a negative pregnancy test result. The exclusion criteria were pregnancy and breast feeding, any serious active disease or comorbid medical condition, any history of another cancer during the last $5 \mathrm{y}$, with the exception of nonmelanoma skin tumors or stage 0 (in situ) cervical carcinoma, and a known hypersensitivity to antibodies or proteins. In the 4 wk preceding immuno-PET, a staging workup that included a complete history, physical examination, CEA, and cancer antigen 15-3 serum level measurements (with biomarker doubling time determination when possible) were performed. The patients underwent morphologic imaging (thoracic-abdominal-pelvic contrastenhanced CT for visceral lesion evaluation and pelvic-spine MRI for bone evaluation) and ${ }^{18}$ F-FDG PET. The trial, sponsored by Nantes University Hospital, was approved by the responsible ethics committee (Comités de Protection des Personnes) and registered at ClinicalTrials.gov (NCT01730612), and all patients gave written informed consent. Enrollment was at the Institut de Cancérologie de l'Ouest, associated with Nantes University Hospital of the health cooperation group of Institut Régional de la Cancérologie Nantes Atlantique.

\section{Investigational Products and Study Design}

The reagents were prepared for human use by Immunomedics, Inc., with a sufficient quantity being produced to complete a small feasibility and safety study. A 1.85-GBq (at calibration time) pharmaceutical-grade ${ }^{68} \mathrm{Ga}$ generator (Eckert-Ziegler) was used. ${ }^{68} \mathrm{Ga}$-IMP288 was obtained with a specific activity of $40-100 \mathrm{MBq} / \mathrm{nmol}$ and a radiochemical purity of more than $95 \%$.

TF2 diluted in $250 \mathrm{~mL}$ of $0.9 \% \mathrm{NaCl}$, and ${ }^{68} \mathrm{Ga}$-IMP288 diluted in $50 \mathrm{~mL}$ of $0.9 \% \mathrm{NaCl}$, were administered by intravenous infusion over $30 \mathrm{~min}$. The patients were premedicated with oral antihistamine the day before TF2 infusion and with intravenous antihistamine (5 $\mathrm{mg}$, dexchlorpheniramine) and corticosteroid (500 mg, hydrocortisone hemisuccinate) $5 \mathrm{~min}$ before infusion of 60-120 nmol of TF2, and 3-6 nmol of ${ }^{68} \mathrm{Ga}-\mathrm{IMP} 288$ were administered $24-30 \mathrm{~h}$ later. Four different conditions of pretargeting (molar doses of BsmAb and peptide and delay) were assessed for pharmacokinetics optimization purposes. Because there were no statistically significant differences among the cohorts, the results for the 4 cohorts were combined to compare the sensitivity of immuno-PET with that of ${ }^{18} \mathrm{~F}-\mathrm{FDG}$ PET or conventional imaging.

Safety was assessed through vital signs, physical examination, and adverse events. HAHA titers were determined by Immunomedics at 3 or 6 mo, and up to 9 mo in some cases, using an enzyme-linked immunosorbent assay (abnormal when $\geq 50 \mathrm{ng} / \mathrm{mL}$ ) (25).

\section{Immuno-PET Imaging}

$\mathrm{PET} / \mathrm{CT}$ was performed using a 4-ring Siemens Biograph mCT system 60 and $120 \mathrm{~min}$ after injection of $150 \mathrm{MBq}$ of ${ }^{68} \mathrm{Ga}$-IMP288 and reconstructed using 3-dimensional ordinary Poisson ordered-subsets expectation maximization with point-spread-function correction and time-of-flight mode ( 3 iterations, 21 subsets, gaussian postfiltering of $2 \mathrm{~mm}$ in full width at half maximum, $4 \times 4 \times 2 \mathrm{~mm}$ voxels). Wholebody acquisitions were performed under spontaneous breathing for 2.5 min per bed position. CT was obtained using variable mAs, 120 $\mathrm{kVp}$, and a pitch of 1 without contrast enhancement. Acquisitions were performed from the top of the head to mid thigh (6-8 steps per patient).

\section{Qualitative Imaging Analysis}

Immuno-PET abnormal uptake was defined visually as a focal increase in uptake higher than the surrounding background level. If more than 10 lesions per bone or more than 10 lesions for the entire liver were counted, the lesion number was capped at 10 . With masking to the other diagnostic results, CT and bone MR images were analyzed by the consensus of 2 radiologists with expertise in oncology, and ${ }^{18}$ F-FDG PET and immuno-PET images were analyzed by the consensus of 2 nuclear medicine physicians with expertise in immunotargeting and PET. For both ethical and practical reasons, not every suspected lesion was evaluated by histology. Complementary imaging, primarily MRI, was performed to assess the most important lesions suspected by immuno-PET and not detected by the initial work-up (CT and bone MRI). The gold standard was therefore determined on the basis of histology and imaging follow-up. Indeed, ${ }^{18}$ F-FDG PET, CT, pelvic-spinal MRI, and any additional imaging were performed 3 mo after immuno-PET to confirm the abnormalities.

True-positive (TP) results were abnormal image findings confirmed by histology or detected by at least 1 other imaging modality and confirmed by follow-up. A negative finding on an imaging method was considered to be false-negative (FN) if positive on 1 other imaging method plus histopathology or by 1 other imaging method 
and confirmed by follow-up. Percentage sensitivity $[(\mathrm{TP} / \mathrm{TP}+\mathrm{FN}) \times$ $100]$ on a lesion basis was calculated for each imaging modality.

\section{Semiquantitative Immuno-PET and ${ }^{18}$ F-FDG PET Analyses}

With a threshold of $40 \%$, tumor $\mathrm{SUV}_{\text {max }}$ and $\mathrm{SUV}_{\text {mean }}$ were measured on tumor foci for immuno-PET and ${ }^{18} \mathrm{~F}$-FDG PET, and the partial-volume effect was corrected. Tumor burden was analyzed using total tumor volume (the sum in cubic centimeters of the volume of each positive lesion) and total lesion activity (tumor volume $\times \mathrm{SUV}_{\text {mean }}$ ) obtained automatically with Oncoplanet software (version 2.0 RC; Dosisoft).

\section{Statistical Analysis}

The continuous variables (tumor $\mathrm{SUV}_{\text {max }}$, tumor $\mathrm{SUV}_{\text {mean }}$, total tumor volume, and total lesion activity) were described by median and interquartile range (IQR). Variable comparisons between ${ }^{18} \mathrm{~F}-\mathrm{FDG}$ PET and immuno-PET were performed by means of the Wilcoxon signed-rank test. All comparisons were 2-sided, with a significance limit of less than $5 \%$. All calculations were made using the Stata SE 13.1 statistical tool (StataCorp LP).

\section{RESULTS}

\section{Patient Characteristics and Adverse Events}

The demographics of the 23 metastatic HER2-negative BC patients, all women, are presented in Tables 1 and 2 . Two patients

\section{TABLE 1}

Patient Characteristics, Part 1

\begin{tabular}{|c|c|}
\hline Characteristic & Data \\
\hline Total number of patients & 23 \\
\hline Median age (y) & 61 (range, 37-80) \\
\hline \multicolumn{2}{|l|}{ Initial tumor classification $(n)$} \\
\hline $\mathrm{T} 1$ & 7 \\
\hline $\mathrm{T} 2$ & 14 \\
\hline $\mathrm{T} 4$ & 2 \\
\hline \multicolumn{2}{|l|}{ Initial stage UICC (n) } \\
\hline Stage I & 6 \\
\hline Stage IIA & 10 \\
\hline Stage I/IB & 5 \\
\hline Stage IIIB & 2 \\
\hline \multicolumn{2}{|l|}{ Histology $(n)$} \\
\hline Ductal & 20 \\
\hline Lobular & 3 \\
\hline \multicolumn{2}{|l|}{ Hormone receptor status ( $n$ ) } \\
\hline $\mathrm{ER}+$ and $\mathrm{PR}+$ & 14 \\
\hline $\mathrm{ER}+$ and $\mathrm{PR}-$ & 7 \\
\hline ER- and PR+ & 1 \\
\hline ER- and PR- & 1 \\
\hline \multicolumn{2}{|l|}{ Histologic grade $(n)$} \\
\hline 1 & 1 \\
\hline II & 19 \\
\hline III & 3 \\
\hline Premenopausal status ( $n$ ) & 2 \\
\hline Postmenopausal status ( $n$ ) & 21 \\
\hline \multicolumn{2}{|c|}{$\mathrm{ER}=$ estrogen receptor; $\mathrm{PR}=$ progesterone receptor. } \\
\hline
\end{tabular}

TABLE 2

Patient Characteristics, Part 2

\begin{tabular}{|c|c|}
\hline Characteristic & Data \\
\hline \multicolumn{2}{|l|}{ Primary tumor treatment $(n)$} \\
\hline \multicolumn{2}{|l|}{ Surgery } \\
\hline Yes & 21 \\
\hline No & 2 \\
\hline \multicolumn{2}{|l|}{ Radiotherapy } \\
\hline Yes & 20 \\
\hline No & 3 \\
\hline \multicolumn{2}{|l|}{ Adjuvant chemotherapy } \\
\hline Yes & 22 \\
\hline No & 1 \\
\hline \multicolumn{2}{|l|}{ Adjuvant hormone therapy } \\
\hline Yes & 22 \\
\hline No & 1 \\
\hline \multicolumn{2}{|l|}{$\begin{array}{l}\text { Number of earlier regimens } \\
\text { (chemo- or hormone therapy) }\end{array}$} \\
\hline 1 & 6 \\
\hline 2 & 1 \\
\hline 3 & 5 \\
\hline$>3$ & 11 \\
\hline Median CEA serum level ( $\mu \mathrm{g} / \mathrm{L})$ & 73.45 (range, 35.1-111.8) \\
\hline $\begin{array}{l}\text { Median cancer antigen 15-3 } \\
\text { serum level (kUI/L) }\end{array}$ & 112.6 (range, $12.4-3,000$ ) \\
\hline $\begin{array}{l}\text { Median CEA doubling } \\
\text { time (mo) }\end{array}$ & 4.95 (range, 0.3-330.1] \\
\hline $\begin{array}{l}\text { Median cancer antigen } \\
15-3 \text { doubling time (mo) }\end{array}$ & 4.75 (range, 0.0-94.1) \\
\hline
\end{tabular}

benefited from a second immuno-PET coupled with a second ${ }^{18} \mathrm{~F}$ FDG PET, accounting for 25 immuno-PET and ${ }^{18}$ F-FDG PET studies in the 23 patients.

No patient experienced an anaphylactic reaction during or after the TF2 BsmAb or hapten infusions. HAHA levels were abnormal in 4 of $25(16 \%)$ patients but normalized within 9 mo.

\section{Imaging Results and Sensitivity}

As shown in a previous study with the same radiopharmaceutical (25), the physiologic distribution of the tracer showed moderate liver and spleen uptake, renal elimination, and presence in the heart and blood vessels due to the circulation of the radiopharmaceutical.

In total, 1,178 lesions were confirmed according to the gold standard: 66 in lymph nodes, 29 in the lungs, 153 in the liver, 919 in bone or bone marrow, and 11 in other sites (1 cutaneous, 3 adrenal, 1 ovarian, 1 mammary, and 5 cerebral). All patients had evidence of metastatic disease on immuno-PET, ${ }^{18} \mathrm{~F}$-FDG PET, CT, and bone MRI (Table 3).

In total, 1,116 foci were detected by immuno-PET, all confirmed as TP according to the gold standard, and 62 lesions were missed (FN lesions), predominantly in lung and bone. All immuno-PET lesions were seen at 60 as well as $120 \mathrm{~min} .{ }^{18} \mathrm{~F}-\mathrm{FDG}$ PET, CT, and bone MRI detected 1,056, 216, and 412 TP lesions, 
TABLE 3

Sensitivity of Immuno-PET, ${ }^{18}$ F-FDG PET, and Conventional Imaging

\begin{tabular}{lllll}
\hline \multicolumn{1}{c}{ Location } & \multicolumn{1}{c}{ Immuno-PET } & \multicolumn{1}{c}{ CT } & 18F-FDG PET & Axial bone MRI \\
\hline Overall & 1,116 of $1,178(94.7 \%)$ & Not applicable & 1,056 of $1,178(89.6 \%)$ & Not applicable \\
Lymph nodes & 61 of $66(92.4 \%)$ & 46 of $66(69.7 \%)$ & 59 of $66(89.4 \%)$ & Not applicable \\
Bone & 881 of $919(95.8 \%)$ & Not applicable & 821 of $919(89.3 \%)$ & 412 of $454(90.7 \%)$ \\
Liver & 149 of $153(97.3 \%)$ & 141 of $153(92.1 \%)$ & 145 of $153(94.8 \%)$ & Not applicable \\
Lung & 14 of $29(48.3 \%)$ & 29 of $29(100 \%)$ & 22 of $29(75.9 \%)$ & Not applicable \\
\hline
\end{tabular}

respectively, and presented 122,35 , and $42 \mathrm{FN}$ results, respectively (Table 3 ). The mammary and cutaneous sites were detected by $\mathrm{CT},{ }^{18} \mathrm{~F}-\mathrm{FDG}$ PET and immuno-PET, whereas CT missed the adrenal lesions and ${ }^{18} \mathrm{~F}$-FDG PET missed the ovarian lesion. Interestingly, immuno-PET detected 5 brain lesions confirmed by brain MRI, whereas brain imaging was difficult by ${ }^{18}$ F-FDG PET (4 FN), because of physiologic cerebral ${ }^{18}$ F-FDG uptake (Fig. 1).

Immuno-PET showed a somewhat higher overall sensitivity (94.7\%) than ${ }^{18}$ F-FDG PET $(89.6 \%)$. Regarding the different metastatic sites, immuno-PET also had a higher sensitivity than CT and ${ }^{18}$ F-FDG PET for lymph node $(92.4 \%$ vs. $69.7 \%$ and $89.4 \%$, respectively) and liver (97.3\% vs. $92.1 \%$ and $94.8 \%$, respectively), whereas sensitivity was lower for lung metastases $(48.3 \%$ vs. $100 \%$ and $75.9 \%$, respectively). Immuno-PET had a slightly higher sensitivity for bone evaluation than MRI or ${ }^{18} \mathrm{~F}-\mathrm{FDG}$ PET (95.8\% vs. $90.7 \%$ and $89.3 \%$, respectively) (Fig. 2).

The median size of liver, lung, and lymph node lesions were $24 \mathrm{~mm}$ (range, 10-103 mm), $10.5 \mathrm{~mm}$ (range, 6-19 $\mathrm{mm}$ ), and $28 \mathrm{~mm}$ (range, 9-43 mm), respectively. Of all the lesions identified by ${ }^{18}$ F-FDG and immuno-PET, 3 (a lymph node lesion, a bone lesion, and a cutaneous lesion) were evaluated by immunohistochemistry. Diffuse and heterogeneous staining $(1+$ to $3+$ intensity) was seen in over $50 \%$ of the carcinoma cells (example of lymph node staining is shown in Fig. 3).

\section{PET Quantitative Metrics}

For determination of immuno-PET SUV, total tumor volume, and total lesion activity, only the 15 patients injected with optimized pretargeting parameters according to the study of Bodet-Milin et al. were considered, because these cohorts represent optimal pretargeting conditions that would be applied if the technique were used in clinical practice (i.e., a BsMAb-to-peptide mole ratio of 20 or 40 and a 30-h pretargeting delay) (25). Tumor uptake did not significantly differ between immuno-PET

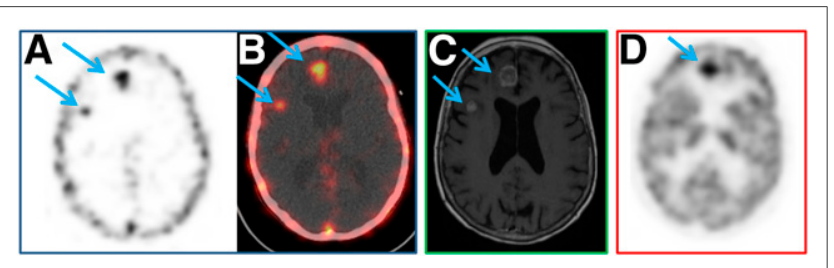

FIGURE 1. (A and B) Pretargeted immuno-PET with TF2 and ${ }^{68} \mathrm{Ga}-$ IMP288 peptide reveals 2 unknown brain metastases (right frontal and right frontoparietal, arrows). (C) T1-weighted gadolinium MR brain image confirms both asymptomatic lesions (arrows). (D) ${ }^{18} \mathrm{~F}$-FDG PET image shows only 1 metastasis (right frontal lesion, arrow). and ${ }^{18}$ F-FDG PET, with a median $\mathrm{SUV}_{\max }$ of 23.83 (IQR, 9.0944.65) and 15.87 (IQR, 11.70-18.87), respectively ( $P=0.088)$, and a median $\mathrm{SUV}_{\text {mean }}$ of 4.90 (IQR, 3.00-8.16) and 4.71 (IQR,: 3.49-5.62), respectively $(P=0.125)$ (Table 4). Tumor burden evaluated by functional volumes was equivalent between the 2 PET methods, with median total tumor volume equal to 294 (IQR, 159-558) for immuno-PET and 299 (IQR, 139-410) for ${ }^{18}$ F-FDG PET $(P=0.256)$, whereas tumor activity total lesion activity was significantly higher with immuno-PET (median of 2,123; IQR, 995-5,304) than with ${ }^{18} \mathrm{~F}-\mathrm{FDG}$ PET (median of 1,197; IQR, 355-2,433; $P=0.009)$. When considering the entire patient population, as described in Figure 4, a correlation was found between the $\mathrm{SUV}_{\max }$ of the most intense lesion in immuno-PET and the serum CEA level $(P=0.0396)$, whereas no correlation was found between ${ }^{18} \mathrm{~F}$-FDG PET tumor $\mathrm{SUV}_{\max }$ and the CEA serum level.

\section{DISCUSSION}

CEA represents a potential target for antibody-based imaging or therapy in several solid tumors, including estrogen receptor-positive, HER2-negative, and triple-negative BC (14-18,26). Moreover, the discrepancy between serum CEA levels and CEA tissue expression in patients with $\mathrm{BC}$ is well known. Whereas immunohistochemistry shows positive CEA expression in 70\%-90\%, serum CEA levels are often within the reference range (16). Further, immunohistochemistry is often performed only on a tumor biopsy sample (i.e., a small fragment of the disease), whereas an antiCEA PET imaging study will allow an evaluation of CEA expression in tumors disseminated throughout the whole body. Indeed, it is possible that an examination of the heterogeneity of CEA expression in the disseminated tumors, as elucidated by imaging, may improve the prediction of antitumor responses. This initial study is the first demonstrating the excellent sensitivity of anti-CEA pretargeted immuno-PET in HER2-negative metastatic BC, suggesting its potential for tumor imaging or theranostic approaches in this subtype of $\mathrm{BC}$ or other CEAexpressing tumors.

In this study, pretargeted immuno-PET achieved a $94.7 \%$ overall sensitivity, with a somewhat better sensitivity than morphologic imaging or ${ }^{18}$ F-FDG PET for lymph node, liver, and bone examinations in $\mathrm{BC}$ patients with metastatic disease. As described in the literature, the number of FN results by ${ }^{18} \mathrm{~F}$-FDG PET may be accentuated by most patients having a hormonal status of estrogen receptor-positive or progesterone receptor-positive (2). However, in contrast to ${ }^{18} \mathrm{~F}$-FDG PET, immuno-PET detected brain metastases, potentially providing improved patient management due to the possibility of theranostic targeting of cerebral dissemination. Disappointingly, immuno-PET was less effective than CT or ${ }^{18} \mathrm{~F}$-FDG PET for 


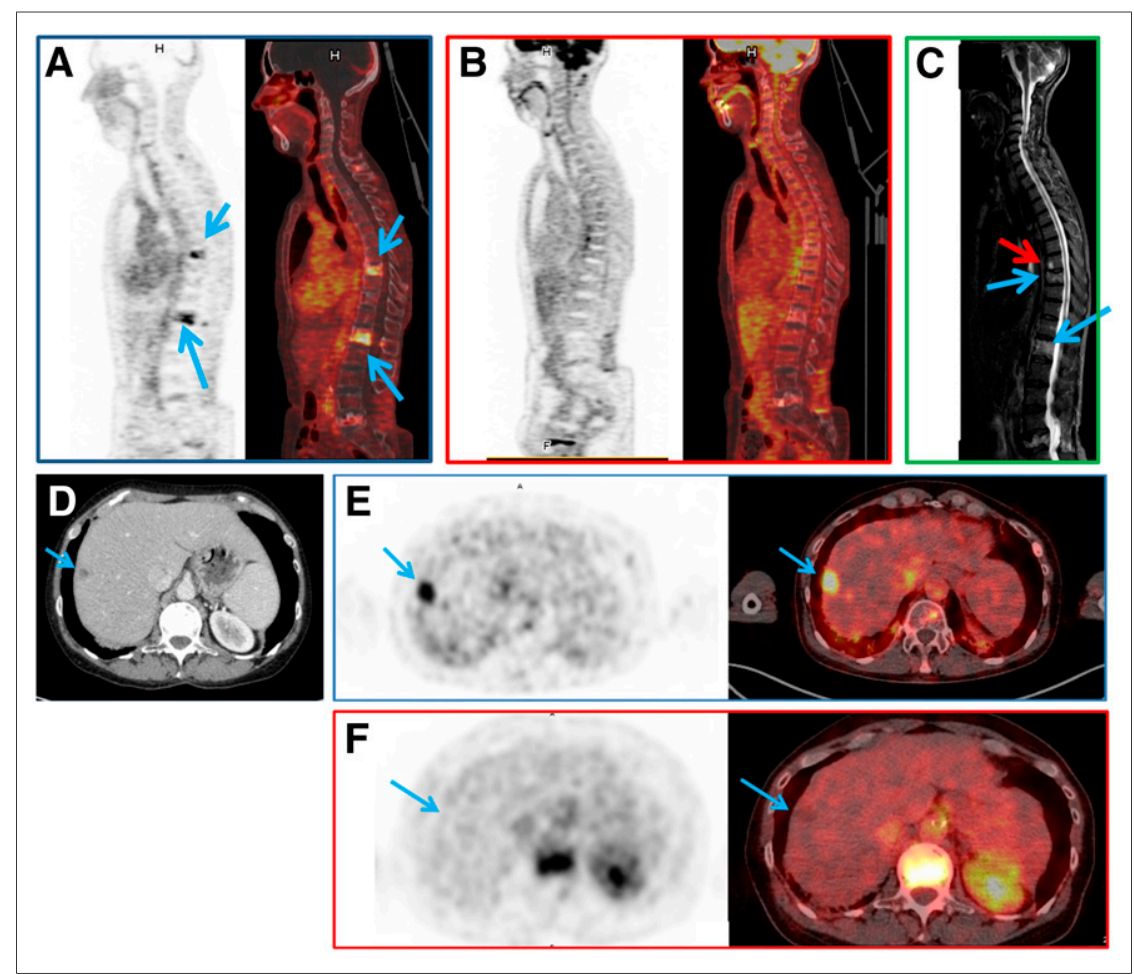

FIGURE 2. (A-C) In patient 1, pretargeted immuno-PET with TF2 and ${ }^{8} \mathrm{Ga}-\mathrm{IMP} 288$ peptide images show 2 vertebral metastases (L1 and T9, arrows) (A), ${ }^{18} \mathrm{~F}$-FDG PET discloses no vertebral abnormalities (B), and vertebral MRI confirms both lesions (blue arrows) and discloses another (red arrow) at T8 (C). (D-F) In patient 2, CT shows suspected liver lesion (D) and pretargeted immuno-PET with TF2 and ${ }^{68} \mathrm{Ga}-\mathrm{IMP} 288$ peptide reveals high uptake by liver lesion (arrow) (E), which was not seen by ${ }^{18} \mathrm{~F}-\mathrm{FDG}$ PET (F).

lung metastasis detection, whereas the sensitivity of CT and ${ }^{18} \mathrm{~F}-\mathrm{FDG}$ PET was consistent with that reported previously $(27,28) .{ }^{68} \mathrm{Ga}-\mathrm{PET}$ has a larger range than ${ }^{18} \mathrm{~F}(6.10$ vs. $0.54 \mathrm{~mm}$ in lung tissue, respectively), reducing PET image resolution, whereas the spontaneous-breathing PET/CT acquisition associated with a partial volume may affect PET performance with ${ }^{68} \mathrm{Ga}$ more than that with ${ }^{18} \mathrm{~F}$ for detection of small lung lesions (median value of $10.5 \mathrm{~mm}$ in this cohort) (29). Moreover, Laessig et al. reported a decreased expression of CEA in lung metastases, compared with liver and bone metastases (30), as may explain the poorer performance of immuno-PET in this region. However, labeling of the peptide with ${ }^{18} \mathrm{~F}$, as described previously, may improve the detection of small lung lesions (31)

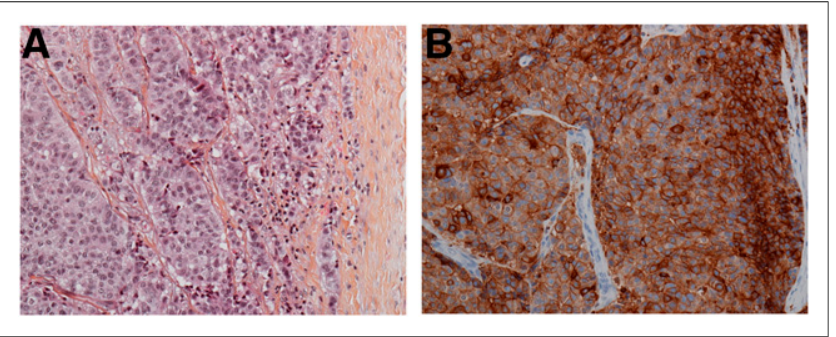

FIGURE 3. A 45-mm left axillary lymphadenopathy excision in patient with initial left ductal BC, $5 \%$ progesterone receptor, $50 \%$ estrogen receptor, and HER2-negative. (A) Carcinoma proliferation surrounded by fibrous capsule (hematoxylin, eosin, and saffron, $\times 200$ ). (B) Diffuse and heterogenous ( + to +++ ) CEA membrane expression in over $50 \%$ of carcinoma cells (immunohistochemical study, $\times 200$ ). without altering radiation exposure, since ${ }^{68} \mathrm{Ga}$ and ${ }^{18} \mathrm{~F}$ have similar exposure rates $\left(1.79 \cdot 10^{-4} \mathrm{mSv} \cdot \mathrm{m}^{2} \cdot \mathrm{MBq}^{-1} \cdot \mathrm{h}^{-1}\right.$ for ${ }^{68} \mathrm{Ga}$ and $1.87 \cdot 10^{-4} \mathrm{mSv} \cdot \mathrm{m}^{2} \cdot \mathrm{MBq}^{-1} \cdot \mathrm{h}^{-1}$ for ${ }^{18} \mathrm{~F}$ ).

CEA, especially CEA cell-adhesion molecule-5, functions as a cell-adhesion molecule during tumor invasion of the lymphatic lumen, possibly explaining the high sensitivity for lymph node detection (32). Indeed, immunohistochemistry confirmed a strong cellular CEA expression in a lymph node showing high uptake in 1 case. Finally, even if imaging specificity requiring histologic confirmation of falsepositive lesions was not determined in this cohort of patients with diffuse disease, immuno-PET is expected to have a higher specificity than ${ }^{18}$ F-FDG PET. This expectation needs further study.

Despite the absence of a significant difference between immuno-PET and ${ }^{18} \mathrm{~F}$ FDG PET for tumor $\mathrm{SUV}_{\text {max }}$ and $\mathrm{SUV}_{\text {mean }}$, which reflect the activity of the most intense lesion, the total lesion activity, reflecting the tumor volume and uptake intensity of all whole-body foci, was significantly higher for immuno-PET than for ${ }^{18}$ F-FDG PET. The total tumor volume, being comparable for the 2 PET methods, suggests that using CEA targeting will result in a higher tumor intensity with whole-body imaging than when using a glucose metabolism tracer, such as in ${ }^{18} \mathrm{~F}-\mathrm{FDG}$ PET.

This initial study also confirmed that intravenous injections of corticosteroids and antihistamines before TF2 and IMP-288 peptide infusions may induce transient immunodepression, limiting immediate and delayed immune reactions. The 16\% HAHA response rate resolved at $9 \mathrm{mo}$ in all cases and was similar to that reported by Bodet-Milin et al. previously and lower than that reported by Schoffelen et al. $(52 \%)(25,33)$.

Finally, immuno-PET with the 2-step pretargeting method described herein enables the use of short-half-life PET emitters, such as ${ }^{68} \mathrm{Ga}$ or ${ }^{18} \mathrm{~F}$, allowing image acquisition only a few hours after peptide injection, whereas using an intact $\operatorname{IgG}$ instead of an antigen-binding fragment requires imaging a few days after the $\mathrm{mAb}$ injection for optimal contrast (because of the delayed immunoglobulin tumor targeting and clearance from the circulation). Intact IgG requires radiolabeling with long-half-life radionuclides, such as ${ }^{89} \mathrm{Zr}$ or ${ }^{124} \mathrm{I}$, which are less favorable in terms of dosimetry. However, pretargeting approaches require optimization studies to determine the best BsmAb and peptide molar dose ratio and pretargeting delay. The TF2 $/{ }^{68} \mathrm{Ga}$-IMP288 system used here has already been evaluated in a study of medullary thyroid cancer patients, concluding that a $120-\mathrm{nmol} \mathrm{BsmAb}$ dose, a molar ratio of 20, and a 30-h pretargeting delay represent optimal conditions (25). The present study on BC patients confirmed the increased tumor uptake and contrast using $120 \mathrm{nmol}$ of BsmAb and a 30-h pretargeting delay, compared with a 60-nmol BsmAb dose or a 24-h pretargeting delay (Supplemental Table 1; supplemental materials are available at http://jnm.snmjournals.org), but this finding requires confirmation in a larger study. 
TABLE 4

PET Semiquantitative Analyses

\begin{tabular}{|c|c|c|c|c|c|}
\hline \multirow[b]{2}{*}{ Parameter } & \multicolumn{2}{|c|}{ Immuno-PET } & \multicolumn{2}{|c|}{${ }^{18} \mathrm{~F}-\mathrm{FDG}$ PET } & \multirow[b]{2}{*}{$P$} \\
\hline & Median & IQR & Median & IQR & \\
\hline Tumor SUV $\max$ & 23.83 & $9.09-44.65$ & 15.87 & $11.70-18.87$ & 0.088 \\
\hline Tumor SUV mean $_{\text {. }}$ & 4.90 & $3.00-8.16$ & 4.71 & $3.49-5.62$ & 0.125 \\
\hline Total tumor volume & 294 & $159-558$ & 299 & $139-410$ & 0.256 \\
\hline Total lesion activity & 2,123 & $995-5,304$ & 1,197 & $355-2,433$ & 0.009 \\
\hline
\end{tabular}

\section{CONCLUSION}

This initial feasibility study demonstrated that anti-CEA immunoPET using a pretargeted trivalent, BsmAb, and then a ${ }^{68} \mathrm{Ga}-\mathrm{IMP} 288$ small peptide is a feasible and safe procedure for detecting metastatic lesions in HER2-negative metastatic BC patients. Immuno-PET targeting CEA showed a higher overall sensitivity than ${ }^{18} \mathrm{~F}$-FDG PET for disclosing metastases, including dissemination in the brain, and thus offers the possibility of an in vivo evaluation of CEA expression and disclosure of whole-body tumor burden, including lesions not accessible to biopsy. Immuno-PET is a potential theranostic molecular imaging technique to select patients for an antibody-based individualized therapy because of metastatic tumor heterogeneity and the discrepancy between serum levels and tissue expression of the antigen in patients.

\section{DISCLOSURE}

David Goldenberg and Robert Sharkey own Immunomedics stock or stock options, and Dr. Goldenberg has royalty-bearing patented inventions. David Goldenberg is the founder and retired chief scientific officer of Immunomedics, Inc., and the founder and retired chairman of IBC Pharmaceuticals, Inc. Robert Sharkey is a consultant to Immunomedics, Inc. The proprietary TF2 and IMP288 reagents were provided by Immunomedics, Inc., and IBC Pharmaceuticals, Inc. This work was supported in part by grants from the University Hospital of Nantes and Fondation Avenir in 2010, the French National Agency for Research, called "Investissements d'Avenir" IRON Labex ANR-11-LABX-001801 and ArronaxPlus Equipex ANR-11-EQPX-0004 and by a grant, INCa-DGOS-Inserm_12558 (SIRIC ILIAD). This work was presented

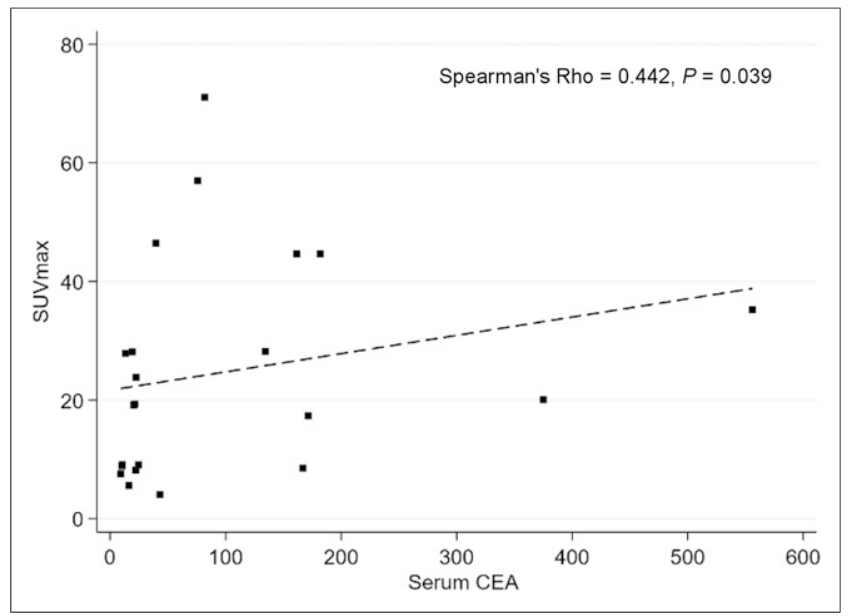

FIGURE 4. Correlation between $\mathrm{SUV}_{\max }$ of most intense lesion in immuno-PET and serum CEA level $(\mu \mathrm{g} / \mathrm{L})$. in part at the 2014 European Nuclear Medicine Congress in Göteborg, Sweden; at the 2014 San Antonio Breast Cancer Symposium in San Antonio, Texas; and at the 2015 annual meeting of the American Society of Clinical Oncology in Chicago, Illinois. No other potential conflict of interest relevant to this article was reported.

\section{ACKNOWLEDGMENTS}

We thank the patients and their families for their participation, and Sabina Belcheva and the nuclear medicine technologists at the Institut de Cancérologie de l'Ouest for their contributions.

\section{KEY POINTS}

QUESTION: Is anti-CEA pretargeting immuno-PET using the BsmAb TF2 and the ${ }^{8}{ }^{8} \mathrm{Ga}-I \mathrm{MP} 288$ peptide a potential theranostic molecular imaging technique to select HER2-negative BC patients for an antibody-based individualized therapy?

PERTINENT FINDINGS: In a prospective clinical trial comparing the sensitivity of anti-CEA pretargeting immuno-PET with ${ }^{18} \mathrm{~F}-\mathrm{FDG}$ PET for 23 HER2-negative metastatic BC patients, we showed a higher overall sensitivity for immuno-PET (94.7\%) than for ${ }^{18}$ F-FDG PET $(89.6 \%)$ for disclosing metastases, particularly in the brain. The possibility is thus offered of an in vivo evaluation of CEA tumor expression, including lesions not accessible to biopsy.

IMPLICATIONS FOR PATIENT CARE: Anti-CEA pretargeting immuno-PET appears to be an efficient theranostic molecular imaging technique for an antibody-based individualized therapy to select patients unable to receive trastuzumab.

\section{REFERENCES}

1. Gerber B, Freund M, Reimer T. Recurrent breast cancer: treatment strategies for maintaining and prolonging good quality of life. Dtsch Arztebl Int. 2010;107:85-91.

2. Salaün PY, Abgral R, Malard O, et al. Good clinical practice recommendations for the use of PET/CT in oncology. Eur J Nucl Med Mol Imaging. 2020;47:28-50.

3. Peterson LM, Kurland BF, Schubert EK, et al. A phase 2 study of $16 \alpha-\left[{ }^{18} \mathrm{~F}\right]-$ fluoro-17 $\beta$-estradiol positron emission tomography (FES-PET) as a marker of hormone sensitivity in metastatic breast cancer (MBC). Mol Imaging Biol. 2014; 16:431-440.

4. de Lucas AG, Schuhmacher AJ, Oteo M, et al. Targeting MT1-MMP as an immunoPET-based strategy for imaging gliomas. PLoS One. 2016;11:e0158634.

5. Jauw YW, Menke-van der Houven van Oordt CW, Hoekstra OS, et al. Immunopositron emission tomography with zirconium-89-labeled monoclonal antibodies in oncology: what can we learn from initial clinical trials? Front Pharmacol. 2016;7:131.

6. Ulaner GA, Lyashchenko SK, Riedl C, et al. First-in-human human epidermal growth factor receptor 2-targeted imaging using ${ }^{89} \mathrm{Zr}$-pertuzumab PET/CT: dosimetry and clinical application in patients with breast cancer. J Nucl Med. 2018;59:900-906. 
7. Rousseau C, Ruellan AL, Bernardeau K, et al. Syndecan-1 antigen, a promising new target for triple-negative breast cancer immuno-PET and radioimmunotherapy: a preclinical study on MDA-MB-468 xenograft tumors. EJNMMI Res. 2011;1:20.

8. Terwisscha van Scheltinga AG, Berghuis P, Nienhuis HH, et al. Visualising dual downregulation of insulin-like growth factor receptor-1 and vascular endothelial growth factor-A by heat shock protein 90 inhibition effect in triple negative breast cancer. Eur J Cancer. 2014;50:2508-2516.

9. Harris L, Fritsche H, Mennel R, et al. American Society of Clinical Oncology 2007 update of recommendations for the use of tumor markers in breast cancer. J Clin Oncol. 2007;25:5287-5312.

10. Molina R, Barak V, van Dalen A, et al. Tumor markers in breast cancer: European Group on Tumor Markers recommendations. Tumour Biol. 2005;26:281-293.

11. Imamura $M$, Morimoto $T$, Nomura $T$, et al. Independent prognostic impact of preoperative serum carcinoembryonic antigen and cancer antigen 15-3 levels for early breast cancer subtypes. World J Surg Oncol. 2018;16:26.

12. Li X, Dai D, Chen B, Tang H, et al. Clinicopathological and prognostic significance of cancer antigen 15-3 and carcinoembryonic antigen in breast cancer: a meta-analysis including 12,993 patients. Dis Markers. 2018;2018:9863092.

13. Böcker W, Schweikhart G, Pollow K, et al. Immunohistochemical demonstration of carcinoembryonic antigen (CEA) in 120 mammary carcinomas and its correlation with tumor type, grading, staging plasma-CEA, and biochemical receptor status. Pathol Res Pract. 1985;180:490-497.

14. Goldenberg DM, DeLand F, Kim E, et al. Use of radiolabeled antibodies to carcinoembryonic antigen for the detection and localization of diverse cancers by external photoscanning. N Engl J Med. 1978;298:1384-1386.

15. Goldenberg DM, Kim EE, De Land FH, et al. Radioimmunodetection of cancer with radioactive antibodies to carcinoembryonic antigen. Cancer Res. 1980;40:2984-2992.

16. Lind P, Smola MG, Lechner P, et al. The immunoscintigraphic use of Tc-99mlabelled monoclonal anti-CEA antibodies (BW 431/26) in patients with suspected primary, recurrent and metastatic breast cancer. Int J Cancer. 1991;47: $865-869$.

17. Goldenberg DM, Abdel-Nabi H, Sullivan CL, et al. Carcinoembryonic antigen immunoscintigraphy complements mammography in the diagnosis of breast carcinoma. Cancer. 2000;89:104-115.

18. Dotan E, Cohen SJ, Starodub AN, et al. Phase I/II trial of labetuzumab govitecan (anti-CEACAM5/SN-38 antibody-drug conjugate) in patients with refractory or relapsing metastatic colorectal cancer. J Clin Oncol. 2017;35:3338-3346.

19. Kalinsky K, Isakoff SJ, Tolaney SM, et al. Safety and efficacy of sacituzumab govitecan (anti-Trop-2-SN-38 antibody-drug conjugate) as $\geq 3$ rd-line therapeutic option for treatment-refractory HER2-negative metastatic breast cancer (HER2Neg mBC). Presented at: San Antonio Breast Cancer Symposium; December 4-8, 2018; San Antonio, Texas. Abstract P2-11-01.
20. Bardia A, Mayer IA, Vahdat LT, et al. Sacituzumab govitecan-hziy in refractory metastatic triple-negative breast cancer. N Engl J Med. 2019;380:741-751.

21. Peltier P, Curtet C, Chatal JF, et al. Radioimmunodetection of medullary thyroid cancer using a bispecific anti-CEA/anti-indium-DTPA antibody and an indium111-labeled DTPA dimer. J Nucl Med. 1993;34:1267-1273.

22. Barbet J, Peltier P, Bardet S, et al. Radioimmunodetection of medullary thyroid carcinoma using indium-111 bivalent hapten and anti-CEA/anti-DTPA-indium bispecific antibody. J Nucl Med. 1998;39:1172-1178.

23. Oudoux A, Salaun P-Y, Bournaud C, et al. Sensitivity and prognostic value of positron emission tomography with F-18-fluorodeoxyglucose and sensitivity of immunoscintigraphy in patients with medullary thyroid carcinoma treated with anticarcinoembryonic antigen-targeted radioimmunotherapy. J Clin Endocrinol Metab. 2007;92:4590-4597.

24. Rossi EA, Goldenberg DM, Cardillo TM, et al. Stably tethered multifunctional structures of defined composition made by the dock and lock method for use in cancer targeting. Proc Natl Acad Sci USA. 2006;103:6841-6846.

25. Bodet-Milin C, Faivre-Chauvet A, Carlier T, et al. Immuno-PET using anticarcinoembryonic antigen bispecific antibody and ${ }^{68} \mathrm{Ga}$-labeled peptide in metastatic medullary thyroid carcinoma: clinical optimization of the pretargeting parameters in a first-in-human trial. J Nucl Med. 2016;57:1505-1511.

26. Waaijer SJH, Warnders FJ, Stienen S, et al. Molecular imaging of radiolabeled bispecific T-cell engager ${ }^{89} \mathrm{Zr}$-Amg2 11 targeting cea-positive tumors. Clin Cancer Res. 2018;24:4988-4996.

27. Yang HL, Liu T, Wang XM, et al. Diagnosis of bone metastases: a meta-analysis comparing ${ }^{18}$ FDG PET, CT, MRI and bone scintigraphy. Eur Radiol. 2011;21:26042617.

28. Piva R, Ticconi F, Ceriani V, et al. Comparative diagnostic accuracy of ${ }^{18}$ F-FDG PET/CT for breast cancer recurrence. Breast Cancer. 2017;9:461-471.

29. Sanchez-Crespo A. Comparison of gallium-68 and fluorine-18 imaging characteristics in positron emission tomography. Appl Radiat Isot. 2013;76:55-62.

30. Laessig D, Nagel D, Heinemann V, et al. Importance of CEA and CA15-3 during disease progression in metastatic breast cancer patients. Anticancer Res. 2007;27:19631968.

31. Schoffelen R, van der Graaf WT, Sharkey RM, et al. Pretargeted immuno-PET of CEA-expressing intraperitoneal human colonic tumor xenografts: a new sensitive detection method. EJNMMI Res. 2012;2:5.

32. Benchimol S, Fuks A, Jothy S, et al. Carcinoembryonic antigen, a human tumor marker, functions as an intercellular adhesion molecule. Cell. 1989;57:327-334.

33. Schoffelen R, Boerman OC, Goldenberg DM, et al. Development of an imagingguided CEA-pretargeted radionuclide treatment of advanced colorectal cancer: first clinical results. Br J Cancer. 2013;109:934-942. 